\title{
KREIN STABILITY IN THE DISTURBED TWO-BODY
}

\section{PROBLEM}

\author{
R. R. CORDEIRO ${ }^{1,2}$ and R. VIEIRA MARTINS ${ }^{1,3}$ \\ 1-Departamento de Astronomia, Observatório Nacional, Rio de Janeiro, Brazil \\ 2-Departamento de Física, Universidade Federal de Viçosa, Viçosa, Brazil \\ (permanent address) \\ and
}

3-Laboratório Nacional de Computação Científica, Rio de Janeiro, Brazil

02 November, 1991

\begin{abstract}
We present a method for the study of the Krein signature in perturbed Hamiltonian integrable systems. The method is developed up to first order in the small parameter. We apply this method to a particular instance of the two-body problem in which the semi-major axis is not affected by the perturbation.
\end{abstract}

Key words: Stability, perturbation, two-body problem.

\section{Introduction}

Physical systems described by Hamiltonians of the form

$$
\mathcal{H}=\sum_{j \geq 0} \varepsilon^{j} \mathcal{H}_{j}
$$

with $0<\varepsilon \ll 1$, are frequently found in Celestial Mechanics. The standard procedure in stability analysis by Krein signatures is to isolate the integrable part, $\mathcal{H}_{0}$, and compute the eigenvalues and eigenvectors of the monodromy matrix (Hadjidemetriou, 1982 and 1985; Howard, 1990). However, when the monodromy matrix for $\mathcal{H}_{0}$ has eigenvalues \pm 1 we need an analysis up to $\mathcal{O}(\varepsilon)$, as in the case of the two-body problem. In this problem we know that many Hamiltonian perturbations do not destroy the stability. This fact suggests that this disturbance leaves the eigenvalues unchanged or transports them to new positions on the unit circle. Therefore, we can have perturbed eigenvalues with the same Krein signatures on the same quadrant of the unit circle so that, by Krein's theorem, any new small arbitrary perturbation can not destroy the stability. But, if the perturbed eigenvalues have different Krein signatures, then a new small arbitrary perturbation may lead to collisions between the eigenvalues, which generates instability.

In the next section we present a method for such analysis and in section 3 it is applied to the two-body problem under a particular Hamiltonian perturbation. 


\section{The Method}

Firstly, we consider a integrable Hamiltonian system given by $\dot{\mathbf{z}}=J D \mathcal{H}_{0}$, with $\mathbf{z} \in \mathcal{T}^{n} \times \mathcal{R}^{n}$ ( $n$ is the degree of freedom and $\mathcal{T}^{n}$ is $\mathrm{n}$-torus), $D \mathcal{H}_{0}$ is the Jacobian of $\mathcal{H}_{0}$, and

$$
J=\left(\begin{array}{cc}
O_{n} & I_{n} \\
-I_{n} & O_{n}
\end{array}\right)
$$

( $I_{n}$ and $O_{n}$ are the $n \times n$ unit and zero matrices, respectively). Allied to the above systems we have the variational equation (or tangent map) of the form

$$
\dot{\xi}=\left(J D^{2} \mathcal{H}_{0}\right)_{z_{\tau}} \xi
$$

where $\left(D^{2} \mathcal{H}_{0}\right)_{z_{\tau}}$ is the Hessian of $\mathcal{H}_{0}$ restrict to a $\tau$-periodic solution, $\mathbf{z}_{\tau}$, supposing it exists. Therefore we have that (2) is a system of linear differential equations with $\tau$-periodic coefficients, as required by Krein's theory (Moser, 1958 ;Yakubovich and Starzhinskii, 1975).

If $\mathbf{z}_{o}=\mathbf{z}_{\tau}\left(t_{o}\right)$ is taking as the initial condition for the integrable problem then (see for example Hirsch and Smale, 1975 , chap. 15) a solution of the equation (2) is

$$
\xi=\frac{\partial \mathbf{z}_{\tau}}{\partial \mathbf{z}_{o}} .
$$

Now we considerer a Hamiltonian perturbation given by (1). The Hamilton's equation are now $\dot{\mathbf{z}}=J D \mathcal{H}_{0}+\sum_{j \geq 1} \varepsilon^{j} J D \mathcal{H}_{j}$, with solution of the form $\mathbf{z}=\mathbf{z}\left(t, \mathbf{z}_{o}^{\prime}\left(\varepsilon, \mathbf{z}_{o}, t\right)\right)$, where $\mathbf{z}_{o}^{\prime}$ is such that for $\varepsilon=0, \mathbf{z}_{o}^{\prime}=\mathbf{z}_{o}$. So we have the new variational equations:

$$
\dot{\xi}=\left(J D^{2} \mathcal{H}_{0}+\sum_{j \geq 1} \varepsilon^{j} J D^{2} \mathcal{H}_{j}\right)_{z_{\tau}} \xi .
$$

Therefore, as in the previous case, $\mathbf{z}_{\tau}$ is a $\tau$-periodic solution, not necessarily with the same period of the integrable system. The existence of such solution is guaranteed, under some conditions, by Poincare's theorem on the families of periodic solutions (Poincaré, 1892, chap 3).

So we have that the solution of (4) is (3), which we can write as

$$
\xi=\frac{\partial \mathbf{z}_{\tau}}{\partial \mathbf{z}_{o}^{\prime}} \frac{\partial \mathbf{z}_{o}^{\prime}}{\partial \mathbf{z}_{o}} .
$$

Let

$$
\mathbf{z}_{o}^{\prime}=\sum_{j \geq 0} \varepsilon^{j} \mathbf{z}_{j}
$$

where $\mathbf{z}_{j}=\mathbf{z}_{j}\left(\mathbf{z}_{o}, t\right)$ with $j \geq 1$, then

$$
\xi=\frac{\partial \mathbf{z}_{\tau}}{\partial \mathbf{z}_{o}^{\prime}}\left[I_{2 n}+\sum_{j \geq 1} \varepsilon^{j} \frac{\partial \mathbf{z}_{j}}{\partial \mathbf{z}_{o}}\right] .
$$


If the condition $\xi\left(t_{o}\right)=I_{2 n}$ does not verify we can multiply $\xi$ by the constant matrix $\left[\xi\left(t_{o}\right)\right]^{-1}$. Thus,

$$
\xi^{\prime}(t)=\xi(t)\left(\xi\left(t_{0}\right)\right)^{-1}
$$

As the matrizant $\xi^{\prime}$ is derived of a Hamiltonian system we have the sympletic condition

$$
\left(\xi^{\prime}\right)^{\dagger} J \xi^{\prime}=J
$$

with $\nmid$ stands for transpose. From (7) and (8), considering the terms until $\mathcal{O}(\varepsilon)$, we have

$$
J \frac{\partial \mathbf{z}_{1}^{\prime}}{\partial \mathbf{z}_{o}}+\left[\frac{\partial \mathbf{z}_{1}^{\prime}}{\partial \mathbf{z}_{o}}\right]^{\dagger} J=0
$$

where $\mathbf{z}_{1}^{\prime}=\mathbf{z}_{1}(t)-\mathbf{z}_{1}\left(t_{o}\right)$. Therefore, if

$$
\frac{\partial \mathbf{z}_{1}^{\prime}}{\partial \mathbf{z}_{o}}=\left(\begin{array}{ll}
A_{1} & A_{2} \\
A_{3} & A_{4}
\end{array}\right)
$$

where $A_{i}(i=1, \cdots, 4)$ are $n \times n$ matrices, from (9) we obtain

$$
\begin{aligned}
& A_{4}=-A_{1}^{\dagger}, \\
& A_{3}=A_{3}^{\dagger}, \\
& A_{2}=A_{2}^{\dagger} .
\end{aligned}
$$

Considering terms of order up to $\mathcal{O}(\varepsilon)$ in $(10)$ and (7) we have

$$
\left(\xi^{\prime}\right)^{\dagger} J \xi^{\prime}=J+\sum_{j \geq 2} \varepsilon^{j} B_{j}
$$

where $B_{j}$ are $2 n \times 2 n$ matrices.

Finally, the eigenvalues, $\lambda$, and eigenvectors, $\mathbf{v}$, are related by

$$
\xi^{\prime}(\tau) \mathbf{v}=\lambda \mathbf{v}
$$

and the Krein signatures associated of the eigenvalue $\lambda$ is the sign of the real number given by indefinite scalar product of the form

$$
<\mathbf{v}, \mathbf{v}>\equiv i(J \mathbf{v}, \mathbf{v})
$$

where $i=\sqrt{-1}$ and $($,$) is the usual scalar product.$ 


\section{Application}

On two-body problem the solution of Hamilton's equation in action-angle variables can be described by Delaunay's variables, here referred as $L_{o i}\left(L_{o}\right.$, $G_{o}$ and $\left.H_{o} ; i=1,2,3\right) \in \mathcal{R}^{3}$ and $l_{o i}\left(l_{o}(t), g_{o}\right.$ and $\left.h_{o} ; i=1,2,3\right) \in T^{3}$. Therefore, by (5) we have that the perturbed variables are

$$
\begin{aligned}
& L_{i}=L_{o i}+\sum_{j \geq 1} \varepsilon^{j} L_{j i}, \\
& l_{i}=l_{o i}+\sum_{j \geq 1} \varepsilon^{j} l_{j i} .
\end{aligned}
$$

Here, we are supposing that $L_{i}(t)$ and $l_{i}(t)$ belong to a $\tau$-periodic solution of a perturbed two-body problem.

Considering (12) up to $\mathcal{O}(\varepsilon)$ and (10), and supposing a particular case where the semi-major axis, $a$, is not affected by the perturbation $\left(L_{1}=\right.$ $\left.L_{o}+\mathcal{O}\left(\varepsilon^{2}\right)\right)$ we obtain

$$
\xi^{\prime}(\tau)=\left(\begin{array}{cccccc}
1 & 0 & 0 & 0 & 0 & 0 \\
\varepsilon \alpha_{21} & 1+\varepsilon \alpha_{22} & \varepsilon \alpha_{23} & 0 & \varepsilon \alpha_{25} & \varepsilon \alpha_{26} \\
\varepsilon \alpha_{31} & \varepsilon \alpha_{32} & 1+\varepsilon \alpha_{33} & 0 & \varepsilon \alpha_{26} & \varepsilon \alpha_{36} \\
\frac{-3 \tau}{a^{2}}+\varepsilon \alpha_{41} & \varepsilon \alpha_{42} & \varepsilon \alpha_{43} & 1 & -\varepsilon \alpha_{21} & -\varepsilon \alpha_{31} \\
\varepsilon \alpha_{42} & \varepsilon \alpha_{52} & \varepsilon \alpha_{53} & 0 & 1-\varepsilon \alpha_{22} & -\varepsilon \alpha_{32} \\
\varepsilon \alpha_{43} & \varepsilon \alpha_{53} & \varepsilon \alpha_{63} & 0 & -\varepsilon \alpha_{23} & 1-\varepsilon \alpha_{33}
\end{array}\right)
$$

where the $\alpha_{i j}$ are

$$
\begin{array}{ll}
\alpha_{i j}=\frac{\partial L_{1 i}}{\partial L_{0 j}}(\tau)-\frac{\partial L_{1 i}}{\partial L_{0 j}}\left(t_{o}\right) & (i, j \leq 3), \\
\alpha_{i j}=\frac{\partial L_{1 i}}{\partial l_{0(j-3)}}(\tau)-\frac{\partial L_{1 i}}{\partial l_{0(j-3)}}\left(t_{o}\right) & (i \leq 3, j>3), \\
\alpha_{i j}=\frac{\partial l_{1(i-3)}}{\partial L_{0 j}}(\tau)-\frac{\partial l_{1(i-3)}}{\partial L_{0 j}}\left(t_{o}\right) & (i>3, j \leq 3), \\
\alpha_{i j}=\frac{\partial l_{1(i-3)}}{\partial l_{0(j-3)}}(\tau)-\frac{\partial l_{1(i-3)}}{\partial l_{0(j-3)}}\left(t_{o}\right) & (i, j>3) .
\end{array}
$$

The eigenvalues of $\xi^{\prime}(\tau)$, calculated with symbolic manipulation program Maple 4.2 are

$$
\begin{aligned}
& \lambda_{1,2}=1, \\
& \lambda_{3,4}=1 \pm \varepsilon \sqrt{f\left(\alpha_{i j}\right)+\sqrt{g\left(\alpha_{i j}\right)}},
\end{aligned}
$$




$$
\lambda_{5,6}=1 \pm \varepsilon \sqrt{f\left(\alpha_{i j}\right)-\sqrt{g\left(\alpha_{i j}\right)}}
$$

where $f\left(\alpha_{i j}\right)$ and $g\left(\alpha_{i j}\right)$ are functions of $\alpha_{i j}(i=2, \cdots, 6$ e $j=1, \cdots, 6)$. The eigenvalues $\lambda_{1,2}=1$ are due to the invariance of the semi-major axis.

When $f\left(\alpha_{i j}\right)$ and $g\left(\alpha_{i j}\right)$ are such that $\sqrt{f \pm \sqrt{g}}$ are purely imaginary, we have $\lambda_{3, \cdots, 6}$ on the unit circle (at least up to $\mathcal{O}\left(\varepsilon^{2}\right)$ ). Thus the correspondent indefinite scalar products, (11), are

$$
\begin{aligned}
& <\mathbf{v}_{3,4}, \mathbf{v}_{3,4}>=-\frac{ \pm \sqrt{|f+\sqrt{g}|}}{\left(k\left(\alpha_{i j}\right)\right)^{2}} P_{3,4}\left(\alpha_{i j}\right) \\
& <\mathbf{v}_{5,6}, \mathbf{v}_{5,6}>=-\frac{ \pm \sqrt{|f-\sqrt{g}|}}{\left(k^{\prime}\left(\alpha_{i j}\right)\right)^{2}} P_{5,6}\left(\alpha_{i j}\right)
\end{aligned}
$$

where $P_{3, \cdots, 6}\left(\alpha_{i j}\right)$ are real functions and

$$
\begin{aligned}
& P_{3,4}=A\left(\alpha_{i j}\right)(f+\sqrt{g})^{2}+B\left(\alpha_{i j}\right)(f+\sqrt{g})+C\left(\alpha_{i j}\right) \\
& P_{5,6}=A\left(\alpha_{i j}\right)(f-\sqrt{g})^{2}+B\left(\alpha_{i j}\right)(f-\sqrt{g})+C\left(\alpha_{i j}\right)
\end{aligned}
$$

Therefore, from (13) and (14) we have that the sign of $\left\langle\mathbf{v}_{3}, \mathbf{v}_{3}\right\rangle$ and $\left\langle\mathbf{v}_{5}, \mathbf{v}_{5}\right\rangle\left(\lambda_{3}\right.$ and $\lambda_{5}$ are in same quadrant) depend on the roots

$$
r_{1,2}=\frac{-B \pm \sqrt{B^{2}-4 A C}}{2 A} .
$$

When only one root, $r_{1}$ or $r_{2}$, is between $(f-\sqrt{g})$ and $(f+\sqrt{g})$ we have $\lambda_{3}$ and $\lambda_{5}$ with different signatures. Unlikely, they have equal signatures.

In the case that $\lambda_{3}$ and $\lambda_{5}$ have different signatures, if a perturbation, varying $f$ and $g$, leaves them to collision $(g=0)$ then $f=r_{1}$ or $f=r_{2}$ provided that a eigenvalue $\lambda_{i}$ with multiplicity $1(g \neq 0)$ has $\left\langle\mathbf{v}_{i}, \mathbf{v}_{i}\right\rangle \neq 0$ (Moser, 1958).

Finally, we can conclude, using the Krein's theorem, that if $r_{1}$ or $r_{2}$ belongs to the interval $(f-\sqrt{g}, f+\sqrt{g})$, then an arbitrary perturbation that induces a collision of the $\lambda_{3}$ and $\lambda_{5}$ produces instability. Otherwise, for $r_{1}$ and $r_{2}$ not belonging to the interval, all small perturbations generate stability. For the probability of collisions of eigenvalues in the unit circle see Howard and Mackay (1987).

\section{Acknowledgements}

The authors gratefully thanks Dr. J. Koiller for his helpful suggestions and assistance. The comments of Dr. T. Stuchi also are appreciated. 


\section{References}

Hadjidemetriou, J. D.:(1982), Celes. Mechan., 27, 305.

Hadjidemetriou, J. D.:(1985), in Resonances in the Motion of Planets, Satellites and Asteroids, Ferraz-Mello and Sessin eds., Universidade de São Paulo - IAG, São Paulo.

Hirsch, M. W. ; Smale, S.:(1974), Differential Equations, Dynamical Systems, and Linear Algebra, Academic Press, New York.

Howard, J. E. ; Mackay, R. S.:(1987), J. Math. Phys., 28(5), 1037.

Howard, J. E.:(1990), Celes. Mechan. Dynam Astron. , 48, 267.

Moser, J. K.:(1958), Commun. Pure Appl. Math., 9, 673

Poincaré, H.:(1892), Les Méthodes Nouvelles de la Mécanique Céleste, Vol. 1, GauthierVillars et Fils, Paris.

Yakubovich, V. ; Starzhinskii, V. M.:(1975), Linear Differential Equations with Periodics Coefficients, Vol. 1, Halsted Press, New York. 